\title{
Assessing Morphological Resilience. Methodological Challenges for Metropolitan Areas
}

\author{
Giovanni Fusco $^{1(\mathbb{( x )})}$ (1) and Alessandro Venerandi ${ }^{2}$ (1) \\ ${ }^{1}$ Université Côte d'Azur, CNRS, ESPACE, Nice, France \\ giovanni.fusco@univ-cotedazur.fr \\ ${ }^{2}$ Université Côte d'Azur, ESPACE, Nice, France \\ alessandro.venerandi@univ-cotedazur.fr
}

\begin{abstract}
Morphological resilience to urban change is the capacity of the form of the physical city to adapt to everchanging social, economic, and technical contexts. It echoes the theory of general resilience in ecology and is not linked to catastrophic events. Complexity theory informs principles of resilient systems that can be applied to urban form. This paper shows how morphological resilience can be assessed in a wide metropolitan area using geoprocessing protocols and available geospatial information. More specifically, a two-step methodology is proposed and tested on the French Riviera. First, spatial units of analysis are identified based on the interconnectedness of the street network, which is a major morphological component in itself. Next, a set of morphofunctional quantitative indicators of resilience is computed for such spatial units, accounting for both internal structure and integration within the wider metropolitan area. Thirteen indicators are selected to describe five different proxies of morphological resilience: diversity, connectivity, redundancy, modularity and efficiency. The paper also presents the preliminary results of morphological resilience assessment in the French Riviera. Urban central areas on the coast generally show more resilient characteristics, while more contrasted patterns emerge in the hinterland.
\end{abstract}

Keywords: Morphological resilience $\cdot$ Urban form $\cdot$ Geoprocessing $\cdot$ French Riviera

\section{Introduction}

The form of the physical city is at the centre of the planning effort and of urban policies by local authorities. When urban expansion attains the size of a whole metropolitan area, local authorities must manage a great variety of urban forms and identify the most appropriate policies for their conservation and/or transformation. In France, for example, master plans have to be established at the metropolitan level, encompassing thus several municipalities and very diverse urban and suburban contexts. In their founding analyses, these plans have to tackle landscape, architectural, environmental and socioeconomic issues related to urban form. 
The context of the present debate in planning is increasingly the uncertainty of urban and metropolitan futures [4]. Well established paradigms of urban development are questioned by the much needed adaptation to a post-carbon society, to socioeconomic and technical changes, to the integration of new metropolitan functions and, at the same time, to changes in lifestyles and to an increased demand for a sense of place. In this context, new issues arise for metropolitan authorities and for planners and urban geographers supporting them. Which urban forms within a vast metropolitan area are more able to adapt to unknown future socio-technical changes? Which forms are intrinsically fragile because they have been optimized only for a given function and are thus not able to cope with change?

In this very context, Feliciotti [10] and Fusco [13] recently proposed the concept of morphological resilience. If cities cannot foresee their future, we can, as of now, assess the potential resilience (or fragility) of their physical forms and prepare them to the challenges of uncertain futures. Researchers at ESPACE have proposed such an assessment to the planning department of the metropolitan authority Nice-Côte d'Azur, in France, within the POPSU Métropoles national program. Assessing the properties of urban forms in a large metropolitan area inevitably implies the use of appropriate geoprocessing algorithms on available geospatial information. This paper thus relates the operationalization of the concept of morphological resilience at the metropolitan scale and the preliminary outcomes obtained in the French Riviera.

The paper is structured as follows. Section 2 provides the theoretical and methodological bases of morphological resilience and its multidimensional nature. Section 3 presents in more detail the methodology for the assessment of morphological resilience and the case study of the French Riviera metropolitan area. Preliminary results are also mapped and briefly presented. Section 4 concludes with a discussion on the proposed methodology and on its future developments.

\section{Morphological Resilience}

\subsection{Theoretical Bases}

Urban morphological resilience is the ability of the city's physical forms to adapt and transform in the presence of urban change, without requiring heavy operations, such as the destruction and reconstruction of entire neighbourhoods. It is the capacity of the physical city to avoid obsolescence (often even early obsolescence) through selforganized processes of adaptation to change. We are not dealing here with resilience to catastrophic events, being them natural (flooding, earthquake, etc.) or anthropogenic (terrorist attack, industrial accident, etc.). This kind of resilience of urban form has already been studied by a rich scientific literature $[1,8,9,25]$. Rather, we are concerned with the potential adaptability and transformability (or, conversely, with the potential fragility) of the present forms of the physical city when confronted with future socioeconomic and technical changes that urban societies constantly produce endogenously [17, 33, 37], for example, in lifestyles, work organization, and use of technology, in the urban space. Recent works on the concept of resilience $[2,33]$ or even on urban resilience [26] fail to recognize the existence of a specific domain of research on morphological resilience, above all when disconnected from disaster risk reduction. 
From a theoretical point of view, urban morphological resilience is rooted in complexity theory [28-30] and in the theory of general resilience of ecosystems first proposed by Holling [16] and later extended to social-ecological systems [6, 12, 17].

Inspired by previous works in ecology and, in particular, by the ones focusing on the structure and functioning of ecosystems, Mehaffy and Salingaros [27] lay down the founding principles of the theory of resilient urban forms. They identify several physical features characterizing resilience, such as interconnectedness of streets, redundant path types (echoing the high interconnectedness and redundancy of trophic chains in ecosystems), and organic/fine-meshed street networks with small blocks (like the ones of medieval towns or the first planned European and North American cities, such as Turin, Barcelona and Philadelphia) as opposed to tree-like street networks or grids with big blocks. Mehaffy and Salingaros also suggest that resilient urban forms should have a great diversity of activities, building types, functions and populations. Contrary to what was prescribed by functionalist urban planning [24], they consider functional and morphological diversity, across different urban scales, a fundamental aspect of resilience in face of unknown urban change. Fragility, on the other hand, is considered to be linked to the sectorization of urban space, typical of modernist/functionalist zoning, (e.g., CBD, residential neighbourhoods, shopping centres, etc.). Accepting diversity and redundancy thus entails renouncing to optimization (e.g., of form with respect to function, of results with respect to means, etc.). Resilient urban form is not optimized for a given function, but it is redundant and its program is open, thus ensuring greater levels of adaptability in face of possible future functional requirements.

As mentioned above, resilience in urban form should apply to a variety of scales, from the macro-form of the whole metropolitan area, to the finer fabrics of each district and the details of urban design at the micro-scale. Great importance has also been recently attributed to the scale of the single plot [7], as it seems to provide a local, autonomous, and fast response to change. Large urban projects, conceived as a whole, for example with buildings laying above the street on a single concrete slab, are typically fragile urban forms, where incremental adaptation through small additions/transformations is very difficult to achieve. As a result, these projects are often doomed to complete demolition and reconstruction, a sort of admission of failure of non-resilient urbanism. Even in resilient urban forms, urban change often implies a pivot scale of response, but tight trans-scalar relations articulate response at all scales. We can thus think of a level of more autonomous response by individual actors at the finest scale (the plot, the building), whereas change of the morphological infrastructure (the street network, the whole plot system) or the building of large facilities need more coordination among urban actors.

\subsection{Operationalizing Morphological Resilience}

A full conceptualization of morphological resilience goes beyond the scope of this paper. The aforementioned delimitation of scope and concepts is nevertheless needed to tackle the challenging task of operationalizing urban resilience [26]. In this respect, Feliciotti et al. [11] re-interpreted the resilience proxies of social-ecological systems [6] through the lens of urban morphology. Resilience is a property of complex systems that 
cannot be directly measured. Analysts must thus resort to resilience proxies, i.e. context-based attributes indirectly inferable to resilience on the bases of resilience theory. Proxies are operational as they are ascertainable through observation. Feliciotti et al. [11] thus identify five resilience proxies for urban form: diversity, connectivity, redundancy, modularity and efficiency. The diversity contributing to morphological resilience is both functional and typo-morphological. Diversity contributes to system adaptability by providing a multiplicity of options in face of change. Connectivity eases flows (of people, goods, services, information, etc.) within a system and across systems. High connectivity tends to be positive for morphological resilience, although interstices of less-connected fragments can be accommodated within a highly connected whole. Redundancy contributes to morphological resilience by offering a multiplicity of choices that ensure the functioning of the system even when change is happening. Modularity is the degree to which a system's components are made up of identifiable smaller components or parts which can be combined to create larger wholes. High modularity, at different scales, ensures the possibility of piecemeal transformation of urban form at the right scale. Efficiency is a controversial proxy, instead. It is not the optimization of form with respect to a given functional program at a given scale. In a complex system, efficiency requires more structural complexity at each and every scale [34]. Form is thus efficient when it shows a scale-free structure, described by a power-law distribution [19, 35], with many small elements, some intermediate and very few large ones.

For each of the proxies mentioned above, Feliciotti [10] then identifies a set of appropriate measurable indicators. More specifically, she proposes two metrics of diversity, 17 of connectivity, two of redundancy, one of modularity, and one of efficiency. Plot heterogeneity is, for example, a metric of diversity and quantifies to what extent plot sizes are different in a spatial unit. Node degree corresponds to the number of street segments connected to each intersection and measures connectivity. Meshedness indicates to what extent the street network in a spatial unit is more similar to a tree-like structure or a grid and is proposed as a measure of redundancy. Feliciotti also uses these metrics to perform a resilience assessment of the different configurations taken by Gorbals, a neighbourhood in Glasgow (UK), throughout the XX century. This work is a fundamental step forward in the operationalization of morphological resilience. However, it shows some limitations: it focuses on one neighbourhood only rather than on a larger study area; it proposes an unbalanced number of indicators for each resilience proxy; finally, it relies on a fixed spatial unit of analysis, even though resilience has multi-scalar properties. In the next section, we propose a methodology that takes inspiration by Feliciotti's work but that, at the same time, tries to overcome some of its limitations. Details of such a methodology are presented next.

\section{Morphological Resilience in Metropolitan Areas}

The main challenge of the present work is to extend the assessment of morphological resilience to a whole metropolitan area. Morphometric and morpho-functional indicators for each resilience proxy must be calculated through geoprocessing algorithms applicable to easily accessible geographical databases. Before that, though, appropriate 
spatial units of analysis must be determined. A brief presentation of the study area and of available data sources will guide these fundamental methodological steps.

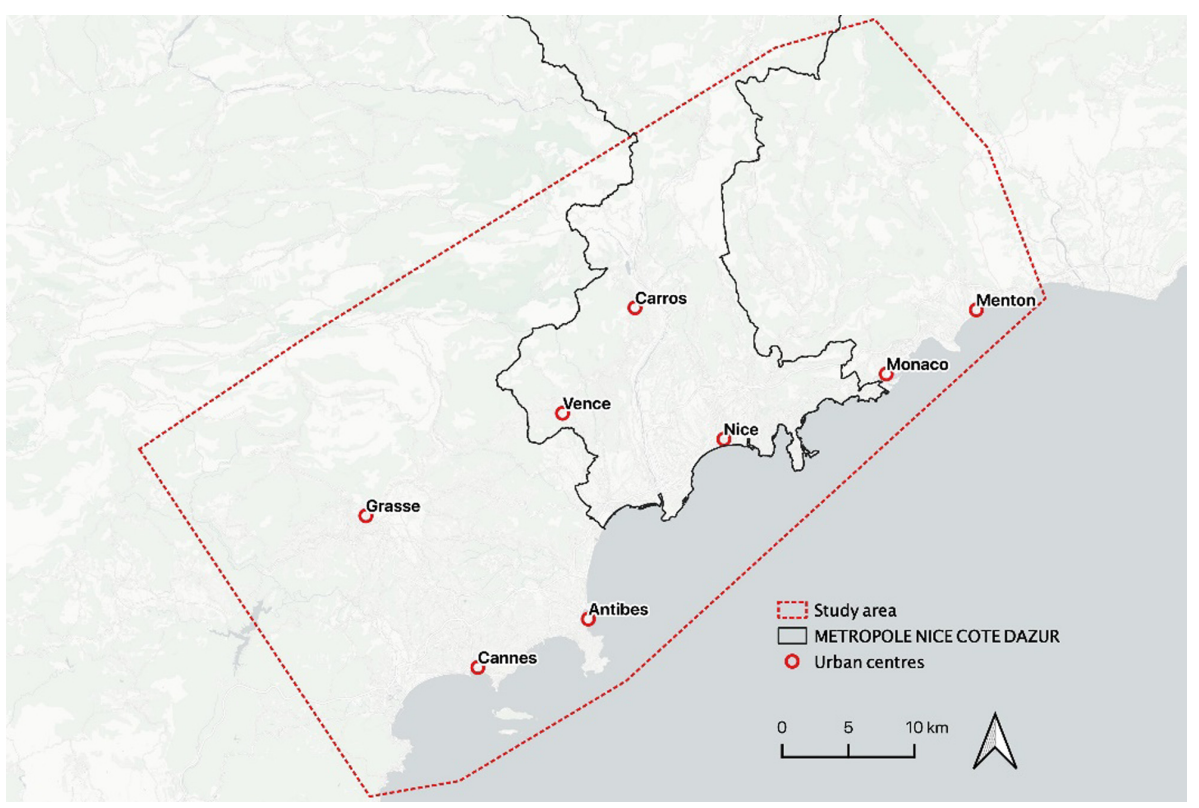

Fig. 1. The French Riviera Metropolitan area and the Metropolitan authority Nice-Côte d'Azur.

\subsection{The French Riviera}

The case study of the analysis is the French Riviera (Fig. 1), an emerging metropolitan area located in Southern France. With a population of more than one million inhabitants over $1,500 \mathrm{~km}^{2}$ [15], this space is characterized by very different urban forms that grew together to form a large conurbation stretching from the French-Italian border (to the east) to the Esterel mountains (closing the bay of Cannes to the west). Such an extensive conurbation developed around the coastal cities of, from east to west, Menton, Monaco, Nice, Antibes and Cannes. Fusco and Araldi [14] reported the existence of nine different types of urban fabric in the French Riviera: old cities and villages, traditionally planned urban fabrics with adjoining buildings, discontinuous and irregular fabrics with houses and buildings, modernist discontinuous fabrics, connective artificial fabrics, two different types of suburban fabrics and two different types of scarcely developed landscapes. These fabrics make up different morphological regions: some are more homogeneous, while others are more mixed (e.g., they are characterized by the co-presence of two or three different fabrics).

The perimeter of the study area does not correspond to any administrative boundary. Although this space forms a unique urban entity, it is in fact fragmented in several local governments, and an independent country (i.e., the Principality of Monaco). More than 
half of its population is nevertheless located in the metropolitan authority of Nice Côte d'Azur (NCA), our partner within the above mentioned POPSU project.

Several geographic databases are available for the French Riviera. The TOPO Database by IGN (the French national cartographic agency) provides a vector representation of buildings, plots and streets with metric precision for the whole of France. Similar data are available on OpenStreetMap for many cities in the world. Urban functions (retail, services, jobs, population counts) are obtained from the database of the local Chamber of Commerce and the household mobility survey. National census and/or local authorities can supply similar data in other world cities.

The methodology presented in this work mainly consists of two steps. The first is the definition of the spatial unit of analysis. The second is the computation of a set of 13 quantitative indicators for such units, describing the five proxies of morphological resilience. We present both next.

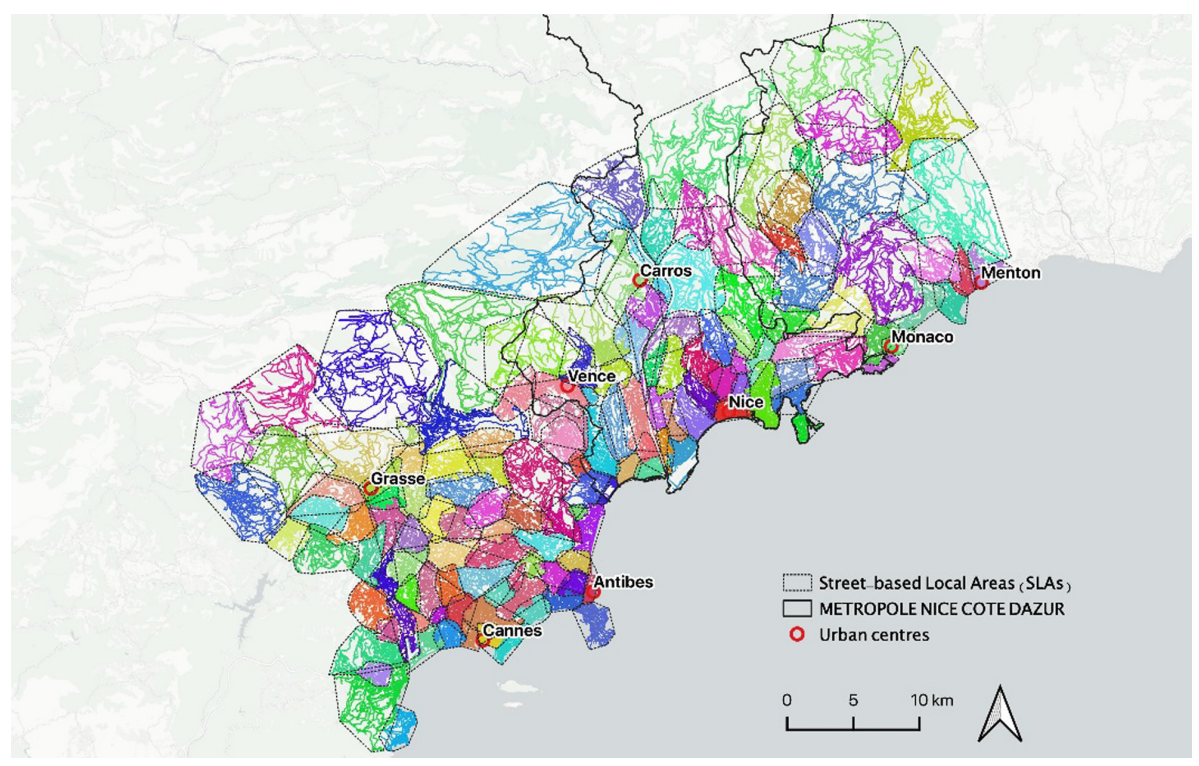

Fig. 2. Partitioning of the French Riviera in Street-based Local Areas (SLAs). Colours are randomly assigned and serve only to make SLAs distinguishable. (Color figure online)

\subsection{Street-Based Local Areas}

The first methodological issue to be addressed is the definition of appropriate spatial units for the analysis. The spatial extent of the assessment is ultimately the whole metropolitan area, making a distinction between its central part (the NCA metropolitan authority) and its eastern (Menton, Monaco, locally named "Riviera") and western (Cannes, Grasse and Antibes) neighbours. Nevertheless, to assess morphological resilience at such a large scale, we first have to identify coherent subspaces of intermediate size to evaluate resilience properties at a more local level. At the same time, 
these units must be big enough to have meaningful assessments of their internal structure. Inter-scalar relationships are fundamental to understand resilience properties. Most of these depend on the links between the micro-scale of plots, buildings and streets and the meso-scale of the spatial units of analysis. Scaling up, relationship between these units and the whole of the metropolitan area also participate to the resilience assessment. The street network is a fundamental component of urban form. It coordinates the organization of plots and buildings at the micro level, but also creates the necessary connections within the morphological system at greater scales. We thus decided to identify intermediate morphological units based on the interconnectedness of the street network. Using the Louvain algorithm of modularity optimization within large networks [3], we thus identified 145 morphological basins, maximizing internal street interconnectedness while minimizing external links. This way of partitioning urban space based on the morphology of the street network has already been applied by Law [23] on the city of London. Law designates the resulting spatial units as Street Local Areas (SLAs). SLAs within the French Riviera typically include between 500 and 800 junction-to-junction street segments. They correspond to urban or suburban districts larger than single neighbourhoods or villages. In the suburbs, they typically include the immediate periphery around village cores. Their limits identify synapses within the street network, i.e. strategic connective segments within the metropolitan area. 52 SLAs fall totally or partially within the administrative area of NCA. A map of the 145 SLAs identified in the French Riviera is presented in Fig. 2. Having defined the basic unit of analysis, we will present next the specific morphometrics for each resilience proxy.

\subsection{Diversity}

Diversity is a fundamental factor for resilience. Both typo-morphological and morphofunctional diversity can be measured for each SLA. Functional diversity is evaluated above all through its primary functional mix, i.e. jobs to residents ratio. The evaluation of this index needs careful attention. Bedroom communities, highly specialized CBDs and commercial areas are all characterized by greater fragility. Ideally, each SLA should have values of jobs to residents ratio similar to the average for the whole metropolitan area. Values smaller than the average tend to be associated with residential areas. Conversely, values greater than the average could at first sight be associated with greater local resilience (as long as jobs are also linked to the presence of other urban functions). Nevertheless, the interconnection among the different SLAs within a whole metropolitan area implies that these above-average values correspond to below-average values elsewhere, hence reducing morpho-functional resilience at the scale of the metropolitan area. For these reasons, the index is calculated as the absolute difference of the jobs to residents ratio from the metropolitan average. The more the SLA differs from the metropolitan average, the more it contributes to morphofunctional fragility.

A second indicator measuring functional diversity focuses on retail and services. The local chamber of commerce identifies 22 different categories of retail and services. Their diversity is a direct contribution to the vitality of the district. We propose to compute such diversity through the Gini-Simpson index [22], which measures the 
probability that two elements randomly chosen within a given SLA are different. Its minimum is zero (in SLAs with only one type of retail or service) its maximum is $1-(1 / \mathrm{k})^{2}$, where $\mathrm{k}$ is the number of different categories (in our case study, these are 22$)$.

The third indicator measures typo-morphological diversity of the buildings within each SLA. As Perez et al. [31] showed for Osaka (Japan) and Marseille (France), a small number of quantitative descriptors can be used to identify types of buildings within a vast metropolitan area. We thus used the very same indicators (i.e., building footprint surface, height, contiguity, convexity index, elongation index and specialization) plus a seventh descriptor (i.e., presence of lightweight extensions, such as verandas, balconies, porches, adjoining sheds, etc.) to cluster the buildings of the French Riviera in types, through a Naive Bayes classifier. After having binned the indicators like in [31], we obtained nine broad types of buildings:

- Type 1. Small compact low-rise detached/semidetached houses or garages.

- Type 2. Detached/semidetached articulated houses with extensions.

- Type 3. Mainly detached articulated small buildings/large villas with extensions.

- Type 4. Small compact adjoining mid-rise buildings and townhouses.

- Type 5. Mid-sized mid-rise relatively compact adjoining buildings.

- Type 6. Mid-sized elongated tall free-standing residential buildings.

- Type 7. Big high-rise articulated and elongated free-standing buildings.

- Type 8. Specialized low-rise compact buildings (sometimes light construction).

- Type 9. Large specialized mid-rise articulated buildings.

The 390000 buildings of the study area were weighted by their footprint area in the clustering algorithm. The optimum number of clusters was determined through a random walk in parameter space under a few constraints (minimum average cluster purity of $90 \%$, maximum of 20 clusters, minimum cluster content of $4 \%$ of building footprint area). The contingency table fit of the selected clustering solution is $58.03 \%$, indicating that the clustering variable accounts for more than half of the information content of the seven original indicators. The diversity of building types is then computed through the implementation of Gini-Simpson index on these nine building classes, in each SLA. Greater values are associated with more morphological resilience, since the presence of different building types allows more flexibility for future functional needs.

Figure 3 shows the Gini-Simpson diversity indexes calculated for the 22 categories of retail and services and the 9 building types, respectively. By visually inspecting the map, we observe that traditional central areas on the coast have relatively greater values of functional diversity, but not necessarily of typo-morphological diversity. The centre of Cannes is an exception and shows lower than expected functional diversity but higher than expected typo-morphological diversity. The hinterland shows smaller values, both in typo-morphological and functional diversity, with few exceptions for the latter. 

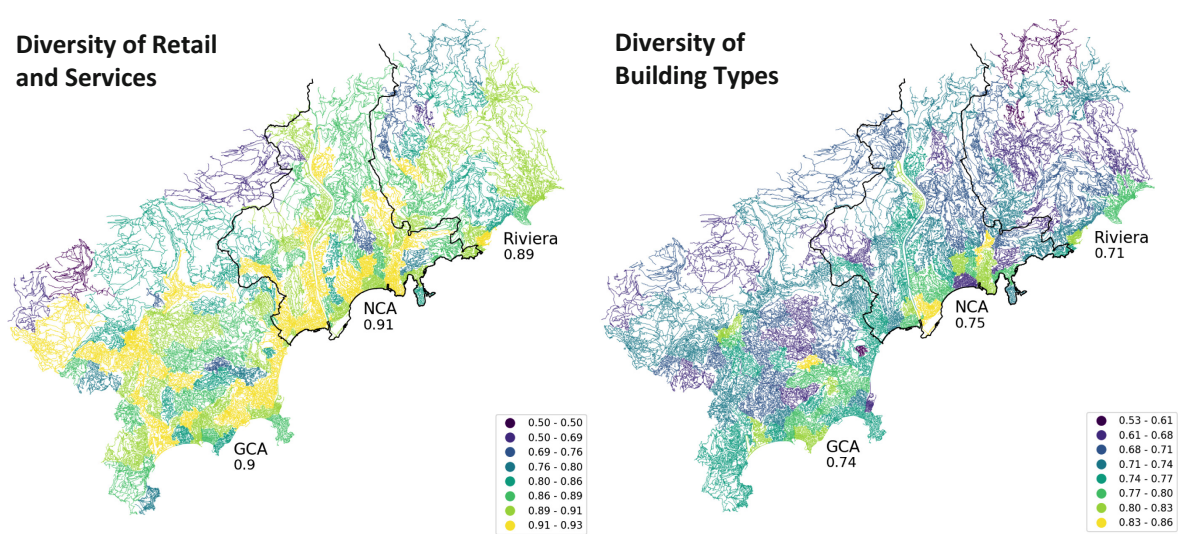

Fig. 3. Gini-Simpson diversity indexes in the SLAs of the French Riviera.
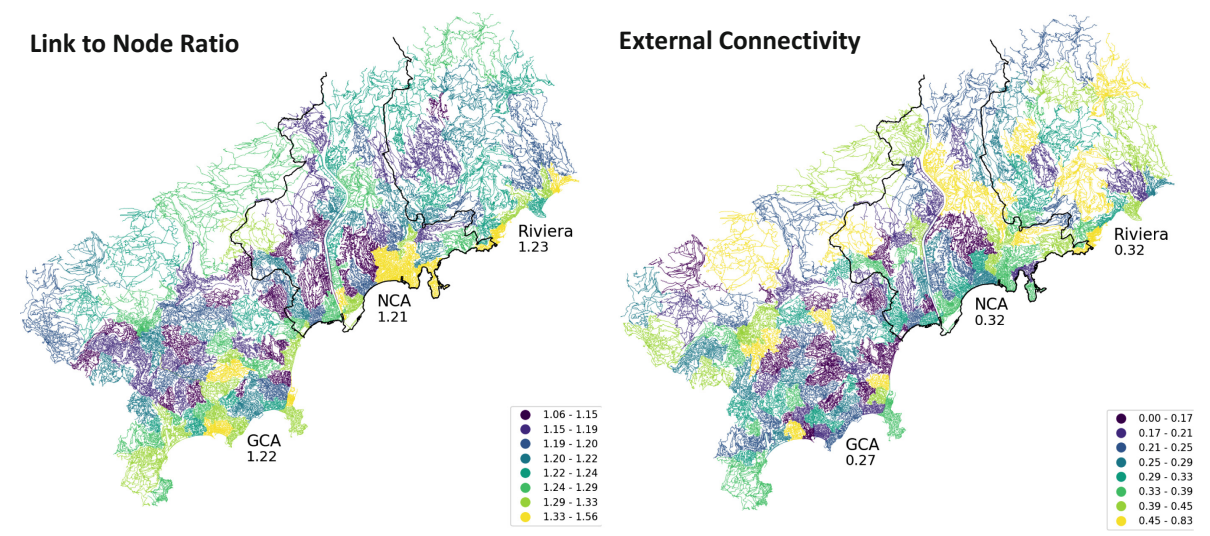

Fig. 4. Street-network connectivity for the SLAs of the French Riviera.

\subsection{Connectivity}

Highly connected systems are more resilient than poorly connected ones [27]. As far as urban form is concerned, connectivity is mainly linked to its street network. This can be described, at the level of SLAs, by classical measures of graph theory: Connected Node Ratio (i.e., the ratio between the number of intersections that are not cul-de-sac and the total number of intersections in each SLA) and Link to Node Ratio (i.e., ratio between the number of links and the number of intersections in each SLA). The spatial units also need to be well connected with the rest of the metropolitan area to ensure resilience at greater scales (and this even though they were identified by minimizing external interconnectedness). We thus measured the External Connectivity of each SLA by computing the ratio between the number of external connections and the square root of the total number of connections in each SLA (which is indicative of the linear dimension of the spatial unit, hence of its perimeter). 
Figure 4 illustrates two of these street-network connectivity indicators. Link to Node Ratio is greater in coastal urban centres. However, the study area does not show a simple coast to hinterland gradient. The smallest values tend to be associated with suburban developments in the close hinterland, especially in the western section of the metropolitan area. Many of such areas are also characterised by low values of outward connections, making them particularly fragile in terms of connectivity. Several peripheral areas in the further hinterland and in the eastern section of the Riviera compensate low Link to Node Ratios with more outward connections.

\subsection{Redundancy}

Redundancy contributes to morphological resilience by offering a multiplicity of choices that ensure functionality in case change happens. In our methodology, this aspect is investigated in terms of urban mobility in two ways: redundancy of paths and multimodality of the transport system. The former is essential both for short-range pedestrian trips and for mid-to-long-range car travel. As already highlighted by Jacobs [18], the possibility of using different paths for daily trips spreads pedestrian and vehicular flows in the city, increases opportunities for services and retail, and avoids charge overload on few axes only [35]. Redundancy of paths is computed through 2 indicators. Path Redundancy assumes that having several streets with good levels of through-movement in each SLA is beneficial. This indicator is computed in two steps. First betweenness centrality [32] is computed for each street in each SLA. Then, we calculate the percentage of streets in the top $50 \%$ of the cumulative distribution of the betweenness values within the SLA. The more segments are needed to attain this threshold, the more resilient the path system is because betweenness centrality is not concentrated in a very small set of street segments.

The second indicator is the Constraint Score of the Path System, i.e. Burt's index [5], which measures to what extents the connections that each street segment establishes with its neighbours are limited within clusters of mutually interconnected segments. SLAs with smaller constraint values tend to have more connections beyond a highly cohesive pool of mutually interconnected segments. This condition can thus be considered positive for morphological resilience. Figure 5 (left) shows that this is usually the case for finely meshed SLAs on the coast (e.g., the city centres of Nice and Antibes) and in the hinterland (e.g., Grasse). Greater values are associated with more fragile areas, for example, the SLAs located in many suburban developments of the close hinterland of the western section of the Riviera.

A different aspect of redundancy is the ability of the network to offer modal alternatives for mid- and long-range trips. The presence of cycle paths, bus routes and high-performance transit service (i.e., light-rail lines and metropolitan railways) were thus considered. Different infrastructures are not independent but operate as multiplex networks working in synergy [36]. The multi-modality potential of the transportation network, named in this work Redundancy of Transport Modes, was computed as the average number of modal options available on the streets of each SLA. To determine the number of modes in each street, we first created buffers around each transport entry point, $300 \mathrm{~m}$ around bus stops and cycle lanes and $600 \mathrm{~m}$ around LRT/railway stations. We then intersected these buffers with each street and added 1 every time a buffer 

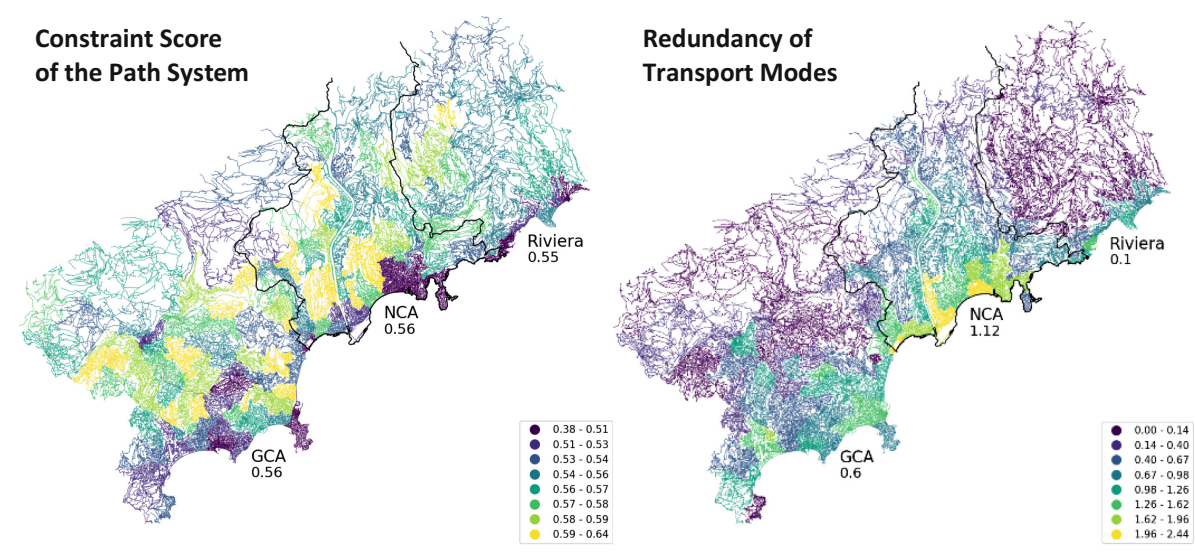

Fig. 5. Redundancy of the mobility system within SLAs of the French Riviera.

corresponding to a new transport mode was found. The minimum value of 0 is associated with areas of car dependence, where private motorized transport is the only available option. The maximum value of 3 is associated with streets with all types of transport modes (i.e., car, buses, cycle lanes, and LRT/railways stations). Figure 5 (right) shows that coastal areas, especially within the NCA perimeter, are characterized by higher redundancy of transport modes, with the exception of the capes. Along with areas in key north-to-south valleys, they benefit from the presence of transportation corridors including roads with dedicated bus lanes, railways and bicycle routes. Most of the hinterland has few alternatives to the car, but this is not the case within the NCA perimeter.

\subsection{Modularity}

Modularity is the degree to which a system's components are made up of identifiable smaller components or parts which can be combined to create larger wholes. High modularity, at different scales, ensures the possibility of piecemeal transformation of urban form at the right scale. Our methodology requires to measure modularity through two indicators, focusing on the plot system and built-fabric.

The plot is the basic component at which scale interventions on urban form are usually carried out. Small plots are easier to be changed/repurposed than larger ones, thus allowing easier bottom-up transformations/adaptations at smaller costs. We propose Plot Granularity as first indicator of modularity. It is computed as the average number of plots per hectare of plots served by the street network. By considering only plots served by street segments, we avoid penalizing SLAs characterized by the presence of undeveloped land. The highest levels of Plot Granularity were found in the coastal cities (Fig. 6, left). A coast-to-hinterland gradient seemed also present, although SLAs representing capes seemed not to follow such a trend.

For what concerned the modularity of the built fabric, the aim was to detect the existence of identifiable modules at different scales, using a geometric sequence of spacing between built-up components. To do so, building footprints were subsequently 
dilated to test whether they could melt in larger structures (i.e., modules) or stayed isolated. This process was performed for the following thresholds: $0 \mathrm{~m}, 1 \mathrm{~m}, 2 \mathrm{~m}, 4 \mathrm{~m}$, $8 \mathrm{~m}, 16 \mathrm{~m}, 32 \mathrm{~m}, 64 \mathrm{~m}$. We then calculated the percentage of built-up components lost at each threshold. Greater percentages at any given dilation indicate that its specific distance is active in structuring built-up modules at SLA level. More precisely, the distance characterising the modules is twice the value of the dilation threshold. The $64 \mathrm{~m}$ dilation, corresponds thus to modules separated by more than $128 \mathrm{~m}$. The $0 \mathrm{~m}$ dilation accounts for the adjoining relations among buildings. This distance is particularly active in continuous built-up fabrics and inactive in discontinuous fabrics of freestanding buildings. The Built-Fabric Modularity indicator thus corresponds to the sum of the percentages of building footprints lost at each of the thresholds mentioned above. The highest values (i.e., between 390 and 447) are associated with the traditional fabric of the coastal cities and the city town of Grasse, in the hinterland. On average, more than $50 \%$ of built-up components are lost at each of the eight dilation thresholds in these areas. Once again, a coast-to-hinterland gradient can be observed (except for Grasse). In SLAs with the smallest values of Built-Fabric Modularity, losses with more than $50 \%$ of built-up components happened rarely (i.e., only for one to three thresholds), meaning that such SLAs show less nested scales in structure of built-up modules.

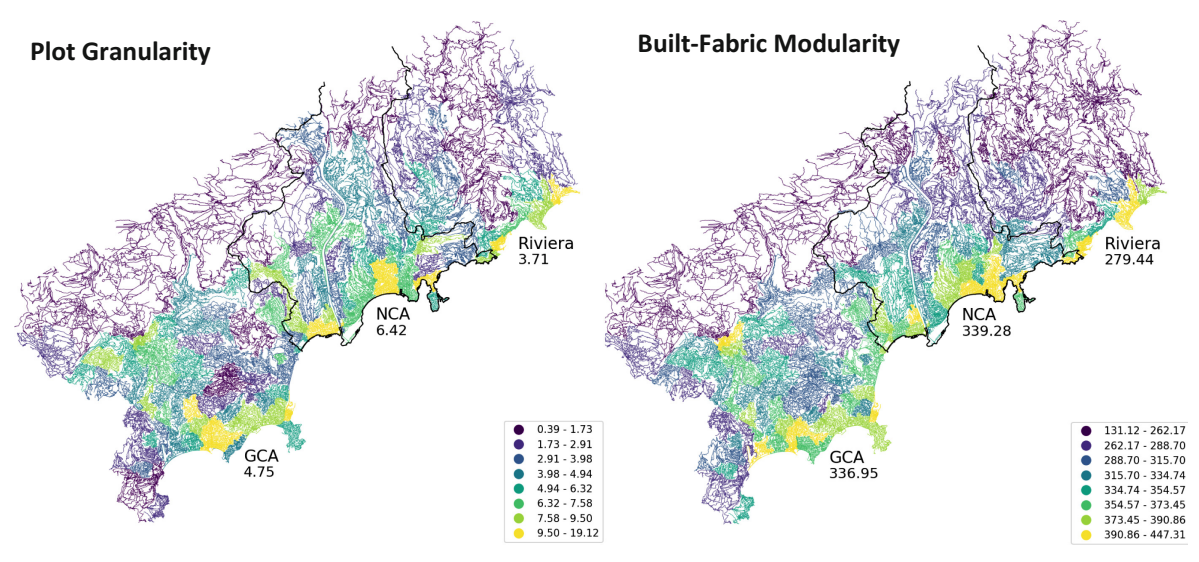

Fig. 6. Morphological modularity in the SLAs of the French Riviera.

\subsection{Efficiency}

Resilient urban form must respect the principles of self-organized complex structures, which proved particularly resilient in natural processes [21, 27]. Efficiency of urban form could thus be measured in terms of degree of self-organized complexity. We propose to measure the morphological efficiency of the built-up fabric and plot system, in each SLA, through the Head/Tail index [19, 20]. The higher the values of such index, the more the empirical distributions of building footprints and plot sizes resemble a power-law distribution. This is a statistical behaviour associated with the self-organization of the morphological system [35], corresponding to the presence of large numbers of small 
elements, several of intermediate size, and very few of big and huge size. In this respect, efficiency is not the contrary of redundancy, but the characteristic of a morphological system to offer all possible dimensional scales, in the way a self-organized system would. Figure 7 shows the Head/Tail indexes of self-organized complexity for plots (Efficiency of the Plot System) and building footprints (Efficiency of the Built-up Fabric), respectively. The two maps show relevant differences. Overall, the plot system shows more complexity levels than the built-up fabric, with most SLAs having 5 to 6 levels of nested complexity and no SLA falling lower than 3. No spatial patterns emerge in this map since small and great values are present everywhere in the metropolitan area under exam. The relative complexity and self-organisation of the plot system is likely to be due to many centuries of both rural and urban history, with the noticeable exceptions of large residential subdivisions in the coastal area. By comparison, the built-up system shows less self-organized complexity and, above all, much more spatial heterogeneity. The coastal cities have the highest levels of complexity (6 or 7), but important sectors of the close hinterland present just 1 or 2 levels of power-law distribution, hinting at processes of control and standardisation of the built-up fabric.

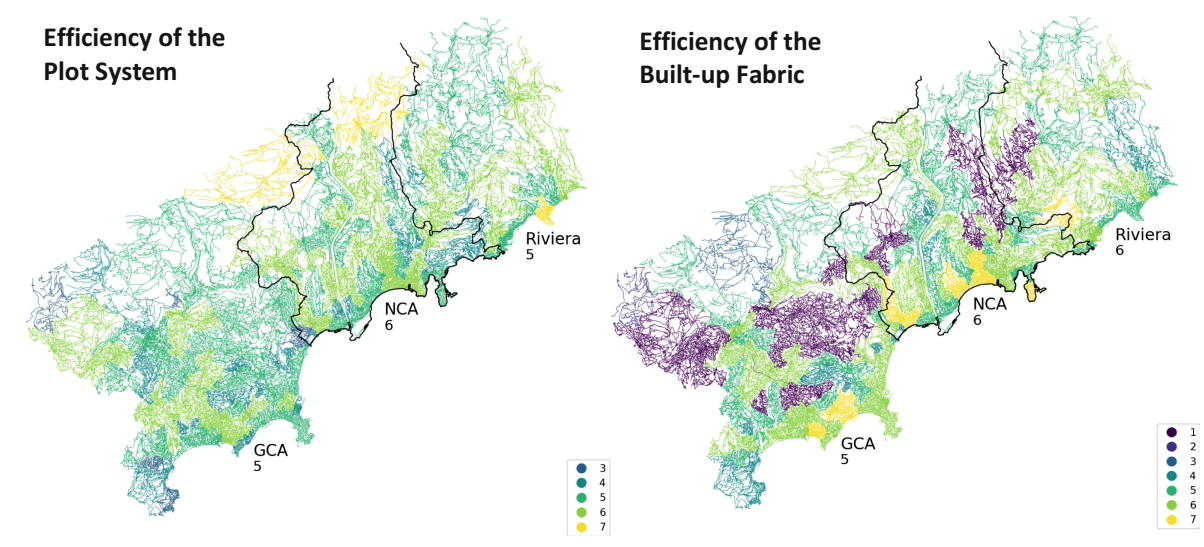

Fig. 7. Efficiency of the morphological system in the SLAs of the French Riviera.

\section{Conclusion}

\subsection{Assessing the Methodological Proposal}

The main goal of this paper was to show how morphological resilience could be assessed in a wide metropolitan area using geoprocessing protocols and available geospatial information. More specifically, we proposed a methodology based on two steps. The first consists in the implementation of the Louvain algorithm of modularity optimization, which identifies spatial units of analysis, the Street-Based Local Areas (i.e., SLAs), through the evaluation of the interconnectedness of the street network of the study area under exam. The second step consists in computing a set of morphofunctional indicators of resilience describing five different proxies of diversity, connectivity, redundancy, modularity and efficiency. 
Table 1 summarises the indicators that were used to quantify the five different dimensions of morphological resilience in each SLA of the metropolitan area under exam. It is a compact set of 13 quantitative indicators, computable from geospatial information, easily available for the case study and for other world cities.

One main specificity of the proposed assessment is the absence of recognized thresholds for the evaluation of proxy indicators. Table 1 only specifies, on a theoretical basis, whether the indicators are positively or negatively associated with morphological resilience. It is thus only possible to assess their relative performances across the SLAs of a metropolitan area. Possible redundancies among indicators do not constitute an issue, since the assessment is based on the evaluation of proxies and not of individual indicators. At the same time, proxies are not completely independent. Path Redundancy, for example, is linked to the connectivity of the street network and Diversity of Building Types is affected by the Efficiency of the Plot System and of the Built-up Fabric. By construction, proxies are different complementary dimensions of urban morphological resilience.

Table 1. List of resilience indicators for urban form.

\begin{tabular}{|c|c|c|}
\hline $\begin{array}{l}\text { Resilience } \\
\text { proxy }\end{array}$ & Indicator & Evaluation \\
\hline \multirow[t]{3}{*}{ Diversity } & $\begin{array}{l}\text { Primary Functional Mix (Absolute Difference of } \\
\text { Jobs/Residents Ratio from Metropolitan Average) }\end{array}$ & - \\
\hline & Diversity of Retail and Services (Gini-Simpson Index) & + \\
\hline & Diversity of Building Types (Gini-Simpson Index) & + \\
\hline \multirow[t]{3}{*}{ Connectivity } & Street Internal Connectivity (Connected Node Ratio) & + \\
\hline & Street Internal Connectivity (Links to Node Ratio) & + \\
\hline & $\begin{array}{l}\text { External Connectivity (External Links/square root number of } \\
\text { connections) }\end{array}$ & + \\
\hline \multirow[t]{3}{*}{ Redundancy } & $\begin{array}{l}\text { Path Redundancy (Share of street segments concentrating half } \\
\text { of point-to-point minimal paths) }\end{array}$ & + \\
\hline & Constrain Score of the Path System (Burt Index) & - \\
\hline & $\begin{array}{l}\text { Redundancy of Transport Modes (average number of modal } \\
\text { alternatives to the car available in each street) }\end{array}$ & + \\
\hline \multirow[t]{2}{*}{ Modularity } & $\begin{array}{l}\text { Plot Granularity (average number of plots per hectare served } \\
\text { by the street network) }\end{array}$ & + \\
\hline & $\begin{array}{l}\text { Built-Fabric Modularity (\% of loss of built-up elements at } 0- \\
1-2-4-8-16-32-64 \text { m dilations) }\end{array}$ & + \\
\hline \multirow[t]{2}{*}{ Efficiency } & Structural Efficiency of the Plot System (Head/Tail Index) & + \\
\hline & Structural Efficiency of the Built-up Fabric (Head/Tail Index) & + \\
\hline
\end{tabular}

The proposed methodology was tested on the French Riviera, a large metropolitan area in southern France. The Louvain algorithm of modularity optimization identified a total of 145 SLAs, corresponding to urban or suburban districts larger than single neighbourhoods or villages. Partial assessments carried out through the different indicators revealed that the most resilient SLAs are located in urban areas on the coast 
(Monaco, Nice, Antibes, Cannes), with only one case located inland (the city of Grasse). These SLAs all share similar characteristics as they offer functional diversity, but not necessarily the highest Diversity of Building Types. Furthermore, they tend to be well connected internally, but also with respect to the surrounding SLAs and offer redundant paths. Finally, their plots and buildings show self-organized complexity. SLAs with lower levels of resilience tend to be inland and mainly characterised by residential areas served by non-redundant/tree-like street networks, and uniform plot and building sizes. While the findings outlined above are location specific, the methodology is replicable and can thus be applied to assess resilience levels in different study areas.

\subsection{Future Work}

The quantitative indicators proposed in this paper allow a first protocol-based analysis of complementary aspects of morphological resilience in a large metropolitan area. However, they still offer a fragmented appraisal of morphological resilience. The most important perspective for our methodology is thus the development of a third step of the analysis. This will consist in a synthetic multi-dimensional assessment of morphological resilience integrating the five proxies of diversity, connectivity, redundancy, modularity, and efficiency. The absence of recognized thresholds and reference levels, already pointed out for the indicators, will be an important issue for this overall assessment. The multi-scalar aspect of morphological resilience can also be further investigated. The proposed methodology relies on a technique for the identification of spatial units of analysis that can be utilised in study areas of different sizes. However, such an eventuality was not tested in this work. Future work might thus apply the proposed methodology to smaller sub-spaces of the study area.

A more in-depth analysis of the outcome of the resilience assessment in the French Riviera, integrating multi-dimensionality and change of scale, will deserve a dedicated paper. Possible sub-spaces of analysis can be the morphological regions identified by Fusco and Araldi [14] in the French Riviera.

Finally, future research endeavours should consider coupling morphological resilience with other aspects of urban resilience, more closely related to human and social capital in urban areas (governance networks, socio-economic dynamics) or to urban functions (mobility, material and energy flows) as proposed by Meerow et al. [26].

Acknowledgements. This research was carried out thanks to a research grant of the POPSUMétropole program of the PUCA (Plan Urbanisme Construction Architecture) French governmental agency.

\section{References}

1. Abshirini, A., Koch, D.: Resilience, space syntax and spatial interfaces: the case of river cities. ITU A|Z 14(1), 25-41 (2017)

2. Alexander, D.: Resilience and disaster risk reduction: an etymological journey. Nat. Hazards Earth Syst. Sci. 13(11), 2707-2716 (2013) 
3. Blondel, V., Guillaume, J., Lambiotte, R., Lefebvre, E.: Fast unfolding of communities in large networks. J. Stat. Mech: Theory Exp. 10, P10008 (2008)

4. Bunting, T., Filion, P. (eds.): Canadian Cities in Transition - Local Through Global Perspectives. Oxford University Press, Oxford (2006)

5. Burt, R.: Structural holes and good ideas. Am. J. Soc. 110(2), 349-399 (2004)

6. Carpenter, S.R., Westley, F., Turner, M.G.: Surrogates for resilience of social-ecological systems. Ecosystems 8(8), 941-944 (2005). https://doi.org/10.1007/s10021-005-0170-y

7. Cozzolino, S.: The (anti) adaptive neighbourhoods. Embracing complexity and distribution of design control in the ordinary built environment. Environ. Plan. B: Urban Anal. City Sci. 47(2), 203-219 (2020)

8. Cutini V.: The city when it trembles. Earthquake destructions, post-earthquake reconstruction and grid configuration. In: Kim, Y.O., Park, H.T., Seo, K.W. (eds.) Proceedings of the 9th International Space Syntax Symposium. Sejong University, Seoul (2013)

9. Cutini, V., Di Pinto, V.: On the slopes of Vesuvius: configuration as a thread between hazard and opportunity. In: 10th Space Syntax Symposium Proceedings, vol. 66, pp. 1-13. UCL, London (2015)

10. Feliciotti, A.: Resilience and urban design: a systems approach to the study of resilience in urban form. Ph.D. thesis, Strathclyde University, Glasgow (2018)

11. Feliciotti, A., Romice, O., Porta, S.: Design for change: five proxies for resilience in the urban form. Open House Int. 41(4), 23-30 (2016)

12. Folke, C.: Resilience: the emergence of a perspective for social-ecological systems analyses. Glob. Environ. Change 16(3), 253-267 (2006)

13. Fusco, G.: Ville, complexité, incertitude. Enjeux de connaissance pour le géographe et l'urbaniste. Habilitation à Diriger des Recherches en Géographie, Université Côte d'Azur, Nice (2018). https://hal.archives-ouvertes.fr/tel-01968002

14. Fusco, G., Araldi, A.: The nine forms of the French Riviera: classifying urban fabrics from the pedestrian perspective. In: Proceedings of the 24th ISUF International Conference: City and Territory in the Globalization Age, Ed. Universitat Politècnica de València, pp. 13131325 (2017)

15. Fusco, G., Scarella, F.: Recompositions territoriales en Provence-Alpes-Côte d'Azur. Analyse croisée par les mobilités quotidiennes et résidentielles. Cybergeo 656 (2013). http:// cybergeo.revues.org/26080

16. Holling, C.S.: Resilience and stability of ecological systems. Ann. Rev. Ecol. Evol. Syst. 4, $1-23$ (1973)

17. Holling, C.S., Sanderson, S.: Dynamics of (dis)harmony in ecological and social systems. In: Hanna, S., et al. (eds.) Rights to Nature: Ecological, Economic, Cultural, and Political Principles of Institutions for the Environment, pp. 57-86. Island Press, Washington (1996)

18. Jacobs, J.: The Death and Life of Great American Cities. Random House, New York (1961)

19. Jiang, B.: Head/tail breaks: a new classification scheme for data with a heavy-tailed distribution. Prof. Geogr. 65(3), 482-494 (2013)

20. Jiang, B.: Head/tail breaks for visualization of city structure and dynamics. Cities 43, 69-77 (2015)

21. Jiang, B.: Living structure down to earth and up to heaven: Christopher Alexander. Urban Sci. 3(3), 96 (2019). https://doi.org/10.3390/urbansci3030096

22. Jost, L.: Entropy and diversity. Oikos 113(2), 363-375 (2006)

23. Law, S.: Defining street-based local area and measuring its effect on house price using a hedonic price approach: the case study of Metropolitan London. Cities 60, 166-179 (2017)

24. Le Corbusier: La Charte d'Athènes. Editions de Minuit, Paris (1957) 
25. Lhomme, S.: Les réseaux techniques comme vecteur de propagation des risques en milieu urbain. Une contribution théorique et pratique à l'analyse de la résilience urbaine. Thèse de doctorat en géographie, Université Paris-Diderot, Paris (2012)

26. Meerow, S., Newell, J., Stults, M.: Defining urban resilience: a review. Landsc. Urban Plan. 147, 38-49 (2016)

27. Mehaffy, M., Salingaros, N.: Towards Resilient Architectures I: Biology Lessons. Metropolismag.com, March 2013. http://www.resilience.org/stories/2013-03-25/towardresilient-architectures-i-biology-lessons/

28. Morin, E.: Introduction à la pensée complexe. Seuil, Paris (1990)

29. Morin, E.: La Méthode, 6 vols. Seuil, Paris (2006). (Collection Opus)

30. Morin, E.: La complexité humaine. Champs Flammarion, Paris (1994)

31. Perez, J., Fusco, G., Araldi, A., Fuse, T.: Identifying building typologies and their spatial patterns in the metropolitan areas of Marseille and Osaka. Asia-Pacific J. Reg. Sci. 4(2020), 193-217 (2019). https://doi.org/10.1007/s41685-019-00127-6

32. Porta, S., Crucitti, P., Latora, V.: The network analysis of urban streets: a primal approach. Environ. Plann. B: Plann. Des. 33(5), 705-725 (2006)

33. Reghezza-Zitt, M., et al.: What resilience is not: uses and abuses. CyberGeo 2012(621), 123 (2012)

34. Salat, S., Bourdic, L.: Urban complexity, efficiency and resilience. In: Morvaj, Z. (ed.) Energy Efficiency - A Bridge to Low Carbon Economy. IntechOpen (2012). https://www. intechopen.com/books/energy-efficiency-a-bridge-to-low-carbon-economy/urbancomplexity-efficiency-and-resilience

35. Salingaros, N.: Principles of Urban Structure. Techne Press, Delft (2005)

36. Strano, E., Shai, S., Dobson, S., Barthelemy, M.: Multiplex networks in metropolitan areas: generic features and local effects. J. Roy. Soc. Interface 12, 20150651 (2015)

37. Voiron, Ch., Dutozia, J.: Anticiper et simuler les dynamiques de changement pour diagnostiquer et améliorer la résilience du système territorial urbain. Risques Urbains 1, 117 (2017) 